\title{
Stability Analysis to Study the Effects of Different Date of Sowing on Grain Yield Performance in Wheat (Triticum sp.)
}

\author{
Santoshkumar Pujer ${ }^{1 *}$, V. Rudra Naik ${ }^{2}$, Suma S. Biradar ${ }^{2}$ and G. Uday ${ }^{2}$ \\ ${ }^{1}$ SRF, AICRP on Wheat, ${ }^{2}$ Department of Genetics and Plant Breeding, \\ University of Agricultural Sciences, Dharwad 580005, (Karnataka), India \\ *Corresponding author
}

\section{A B S T R A C T}

Key w o r d s
Wheat, Stability,
G×E interaction,
Three dates of
sowing and grain
yield

Genotype $\mathrm{x}$ Environment interaction effects and the stability for grain yield was determined by evaluating eighteen wheat genotypes in three different sowing dates at AICRP on wheat, MARS, University of Agriculture Sciences, Dharwad (Karnataka) during rabi 2017-18 under irrigated conditions using a RCBD with two replications. The genotypes NIAW 34, HW 1098 and BMZ 1516-2 showed higher grain yield $(2965.7,3131.5$ and $3037.7 \mathrm{~kg} / \mathrm{ha})$, average responsiveness $(\mathrm{bi}=1)$ and non-significant $\mathrm{S}^{2}$ di value which suggesting suitability of these genotypes for different dates of sowing. The genotype HW 1098 exhibited superior performance for yield contributed by high tiller under early sown conditions. The genotype HD 3090 recorded lower mean value with average responsiveness (bi=1) indicating, poor adoptability to different dates of sowing and suitable only for timely sowing. The genotypes, GW 322, UAS 415 and DDK 1029 showed higher mean values and bi $>1$ indicating sensitive to environmental changes and specific adaption to early sowing. The genotypes BMZ 15-16-5 shows above average mean value and bi $<1$ indicated specific adoption to only early sowing conditions. Results inferred that the genotypes namely NIAW 34, HW 1098 and BMZ 15-16-2 suitable for all the three dates of sowing condition. It was also found that early sowing is the most optimum time for sowing of wheat crop.

\section{Introduction}

Wheat (Tritium aestivum L.) is the second most important crop that contributes significantly to the global food and food security (Singh, 2013). At global level, wheat occupies an area of $221.68 \mathrm{mha}$, with a production and productivity of $728.28 \mathrm{mt}$ and $32.9 \mathrm{q} /$ ha respectively. India's share in world wheat area is about $12.50 \%$, whereas it occupies $12.05 \%$ share in the total world wheat production, which is the second largest after China (USDA, 2016). In India wheat occupies an area of about 31.23 mha with a production of $98.38 \mathrm{mt}$. and average productivity of $32.16 \mathrm{q} / \mathrm{ha}$ (Indian Institute of

Wheat and Barley Research Annual Report, 2017-18). Information about phenotypic stability is useful for selection of crop varieties in a breeding program. Plant breeders encounter genotype $\times$ environment 
interaction $(\mathrm{G} \times \mathrm{E})$ when testing varieties across the different date of sowing. The magnitude of the interaction or the differential genotypic responses to environments differs greatly across environments (Kaya et al., 2002). The task of breeder is to screen out genotype planted at different interval to enable selection of those varieties, which are suitable for wider range of planting. Hence a study of genotype $\mathrm{x}$ environment interaction can lead to successful evaluation of wheat cultivars for stability in yield performance across environments. The measure of the relative performance of varieties under different environments provides information on stability pattern of these varieties.

\section{Materials and Methods}

Eighteen wheat genotypes viz., UAS 347, NI 5439, GW 322, UAS 304, HI 944, NIAW 34, HD 3090, UAS 415, UAS 428, DDK 1025, DDK 1029, HW 1098, BMZ 15-16-10, BMZ 15-16-5, BMZ 15-16-9, BMZ 15-16-2, BMZ 15-16-7, BMZ 15-16-6, were evaluated at three different dates of sowing (October 25, November 25 and December 25) at AICRP on wheat, MARS, University of Agriculture Sciences, Dharwad (Karnataka) during rabi 2017-18 for twelve characters viz., days to anthesis, NDVI at anthesis, canopy temperature at anthesis, chlorophyll at anthesis, days to maturity, spike length, number of spikelets, tillers per meter, grain yield, biomass, 1000 grain weight and spike length under irrigated conditions using a RCBD with two replications.

GE interaction was analyzed using linear regression techniques. Stability parameter was estimated using the Eberhart and Russell (1966) method. Two stability parameters i.e., regression coefficient (bi) and deviations from regression $\left(\mathrm{s}^{2} \mathrm{di}\right)$ were worked out and tested by using t-test and F-test separately from the pooled analysis.

\section{Results and Discussion}

Pooled analysis of variance for grain yield and yield related components across four environments are presented in the Table 1 following Eberhart and Russell (1966) model. The results revealed that there were significant differences among the genotypes tested at both 5 and 1 per cent level of significance for all the characters studied. The environment in which all the observations were recorded also differed significantly (both at 5 and $1 \%$ probability) to influence significant variation in all the characters recorded.

The mean squares due to genotypes were highly significant against pooled error as well as pooled deviation for various characters except for 1000 grain weight under study indicating sufficient genetic variability present among the genotypes.

Environmental mean squares were also highly significant except spike length and number of spikelets against pooled error and pooled deviation which indicated that environments chosen in the study were highly variable. Significance of E+ (G x E) interactions mean square for most of the characters except days to anthesis, spike length and number of spikelets, tiller/m, 1000 grain weight and grains/spike against pooled error and for all the characters against pooled deviation indicated presence of $\mathrm{G} \times \mathrm{E}$ interaction.

Mean square due to genotypes $\mathrm{x}$ environment (linear) tested against pooled error were significant for three characters viz., CT at anthesis, grain yield ( $\mathrm{Kg} / \mathrm{ha})$ and biomass $(\mathrm{Kg} / \mathrm{ha})$. But were highly significant for all the characters when tested against pooled deviation. This indicated the preponderance of linear component of $\mathrm{G} \times \mathrm{E}$ interaction was higher than non-linear component hence, predictions appeared possible. 
Environment (linear) were significant for all the characters except spike length and number of spikelets indicating significant differences among genotypes in four environments. In genotypes $\mathrm{x}$ environment (linear) portion was higher in magnitude than non-linear (pooled deviation) for CT at anthesis, grain yield (Kg/ha) and biomass ( $\mathrm{Kg} / \mathrm{ha})$, except for days to anthesis, NDVI at anthesis, chlorophyll at anthesis, days to maturity, spike length, number of spikelets, tiller/m, 1000 grain weight and grains/spike.

\section{Days to anthesis}

The studies on estimate of stability parameters revealed that none of the genotype was stable for all the characters. The genotype BMZ 15-16-2 shows stable characters which had showed bi value close to unity $\left(b_{i}=1\right)$ and non-significant $\left(\mathrm{S}^{2} \mathrm{di}\right)$ indicating its superiority for average response and stability over all environment whereas, NI 5439, GW 322, HD 3090, UAS 428, DDK 1029, BMZ 15-16-10 and BMZ 15-16-7 were suitable for favourable environment conditions and the genotype, HW 1029 showed bi value close to unity $\left(b_{i}=1\right)$ and non-significant (s2di) and lower mean value indicating that poorly adapted to all environmental condition for days to anthesis. The results are in agreement with Gulzar et al., (2015), Thakare et al., (2014), Yadava (2003) who reported significance of both linear and non-linear components and indicated the presence of both predictable and unpredictable components of $\mathrm{G} \times \mathrm{E}$. Thakare et al., (2014) and Kashte (2013) has reported the predominance of linear and non-linear components which are in agreement with the present findings.

\section{NDVI at anthesis}

Among stable genotypes, the genotypes, DDK 1025, HW 1098, BMZ 15-16-10 and BMZ
15-16-5 were found to have high mean value and average response $\left(b_{i}=1\right)$ indicating their adoptability over all the environments. The genotype BMZ 15-16-9 with below average responsiveness indicated that specific adaption to favorable environmental condition. The genotypes NAIW 34, UAS 415, DDK 1029 and BMZ 15-16-7 recorded high mean value, bi< 1 and significant $\mathrm{S}^{2}{ }_{\mathrm{di}}$ values indicated the presence of non-linear portion of $\mathrm{G} \times \mathrm{E}$ interaction, which makes it specifically adapted to unpredictable under varying environment conditions.

Remaining all the genotypes was found to be unadapted to tested environments. The genotypes viz., GJHV 500 and F 2177 were found to have earliness and average response $\left(b_{i}=1\right)$ indicated their adoptability over all the environments. The genotype GW 322 shows average responsiveness and lower mean value indicating that poorly adapted to all environmental conditions.

\section{CT at anthesis}

The out of eighteen genotypes five genotypes viz., GW 322, HD 3090, UAS 415, UAS 428 and BMZ 15-16-6 possessed above average mean with $b_{i}>1$ indicating their suitability to favourable environments. The genotype $\mathrm{HW}$ 1098 shows higher average mean value and non-significant for both $b_{i}$ and $S_{d i}^{2}$ indicating suitability for unfavourable environmental condition.

\section{Chlorophyll at anthesis}

The genotypes, BMZ 15-16-10 and BMZ 1516-7 had above average mean values with $b_{i}>$ 1 indicated Specific adaption to favorable environments. The genotypes, NI 5439, UAS 415 and BMZ 15-16-9 recorded highest mean value with above average responsiveness $\left(b_{i}<1\right)$ indicated it's specific adaption to unfavorable environments. 


\section{Days to maturity}

The genotype BMZ 15-16-10 exhibited higher mean value and $b_{i}=1$ indicating their average stability over all environments. The lines viz., UAS 415 and UAS 428 had high mean value with regression coefficient less than unity indicating their adaptability to tested environments. Whereas, the genotype, BMZ 15-16-9 recorded above average mean value for this character with $b_{i}>1$ indicating that these are sensitive to environmental changes but adapted to favourable environments. The genotype HW 1098 shows average responsiveness and lower mean value indicating that poorly adapted to all environmental conditions.

\section{Spike length (cm)}

The genotype, BMZ 15-16-6 depicted above average mean value and $b_{i}>1$ indicating their specific adaptability to favorable environments. The genotypes UAS 304, BMZ 15-16-10, BMZ 15-16-9 and BMZ 15-16-7 shows above average mean value and $b_{i}<1$ indicated specific adoption to unfavourable environmental conditions. These results are in agreement with those obtained by Menshawy (2007).

\section{Number of spikelets}

Genotypes HD 3090, BMZ 15-16-7 and BMZ 15-16-6 depicted above average mean value and $b_{i}>1$ indicated their specific adaption to favorable environments. The genotypes $\mathrm{GW}$ 322, UAS 304, BMZ 15-16-10 and BMZ 1516-2 shows above average mean value and $b_{i}<1$ indicated specific adoption to unfavourable environmental conditions.

\section{Number of tillers/m}

The out of eighteen genotypes, DDK 1029 and HW 1098possessed above average mean with $b_{i}>1$ indicating their suitability to favourable environments. The genotypes viz.,BMZ 15-16-9, and BMZ 15-16-2 shows higher average mean value and nonsignificant for both $b_{i}$ and $S^{2}{ }_{d i}$ indicating suitability for unfavourable environmental condition The results are in agreement with Gulzar et al., (2015), Thakare et al., (2014), Yadava R. (2003).

\section{Grain yield (kg/ha)}

As regard of grain yield, genotypes as well as genotypes and environment interaction was non-significant, indicating no genetic difference among genotypes for environmental response. The genotype BMZ 15-16-9 with highest mean value $(3255.33 \mathrm{Kg} / \mathrm{ha})$ showed the presence of only non-linear portion of G X E interaction which makes its performance unpredictable under varying environments. Among three stable genotypes viz., NIAW 34, HW 1098 and BMZ 15-16-2 possessed above average mean value with regression coefficient $b_{i}=1$ indicating their adoptability to different environments Similar trends have been reported in other multi-locations or multi environments field experiments by Yan et al., (2010) and Rakshit et al., (2012), Motamedi et al., (2012), Kant et al., (2014), Thakare et al., (2015), Lodhi et al., (2015). HD 3090 were found to have low mean value and average response $\left(b_{i}=1\right)$ indicating their poorly adoptable to all the environmental conditions. Similar findings were also reported by Gowda et al., (2010), Meena et al., (2014), Singh and Tyagi (2014) and Kumar et al., (2014). The genotypes GW 322, UAS 415 and DDK 1029 recorded high mean values with above average response $\left(b_{i}>1\right)$ indicating their suitability to favourable environments. The genotype, BMZ 15-16-5 possessed $b_{i}<1$ and above the mean value indicated that specific adoption to unfavourable environmental conditions. 


\section{Biomass (kg/ha)}

The genotypes NI 5439, GW 322, UAS 304, UAS 415, DDK 1029 and HW 1098 depicted above average mean value and $b_{i}>1$ indicating their specific adaptability to favorable environments. The genotypes NI 5439 with high biomass $(\mathrm{kg} / \mathrm{ha})$ significant responsiveness $\left(b_{\mathrm{i}}>1\right)$ indicating that adoptability to favorable environments.

The genotypes HD 3090 and UAS 428 possessed below average mean and bi $=1$ indicating poorly adaptability to all the environments. The genotype BMZ 15-16-10 depicted above average mean value and $b_{i}=1$ indicated well adaptability to all the environments. The genotypes BMZ 15-16-5, BMZ 15-16-7 and BMZ 15-16-6shows above average mean value and $b_{i}<1$ indicated specific adoption to unfavourable environmental conditions.

\section{0 grain weight $(g)$}

The joint regression analysis (Table 1) showed t highly significant GE interaction. The heterogeneity of regression Ms was not significantly against the error, whereas, the remainder from regression Ms was highly significant indicating that non-linear component of GE interaction was operating.

Kaya et al., (2002) reported that there were significant differences between wheat genotypes as well as GE in yield and yield components; a genotype with the lowest or non-significant deviation from regression being the most stable. The only one genotype, viz., HD 3090 has higher mean value, average responsiveness $\quad\left(b_{i}=1\right)$ indicating their adoptability to all the four environments. Similar finding was reported by Meena et al., (2014) and Kumar et al., (2014). The genotype DDK 1025 showed significant regression coefficient deviating from unity indicating unpredictable performance of these genotypes across the environment. The other set of genotypes viz., UAS 347, NI 5439, GW 322, HI 944, UAS 428, DDK 1029, BMZ 1516-5, BMZ 15-16-2, BMZ 15-16-7 and BMZ 15-16-6 were found to be unstable for expression of this trait.

The genotype, NIAW 34 recorded lower mean value with average responsiveness $\left(b_{i}=\right.$ 1) indicating, poorly adoptable to all the four environments. The genotypes, UAS 415 were found having high mean value and $b_{i}<1$ indicated specific adaption to unfavourable environmental conditions.

The genotypes, UAS 304, HW 1098 and BMZ 15-16-10shows higher mean value and $b_{\mathrm{i}}>1$ (below average) indicated specific adaption to favorable environments. Similar findings were reported by Sharma et al., (2012), Swami (2012), Arain et al., (2011), Al-Otayk (2010) Aydin et al., (2010) Kirigwi et al., (2004) and Sial et al., (2000).

\section{Grains/spike}

The genotype, BMZ 15-16-6 exhibited higher mean value and $b_{i}=1$ indicating their average stability over all environments. The lines viz., UAS 347, NI 5439, BMZ 15-16-5 and BMZ 15-16-2 had high mean value with regression coefficient less than unity indicating their adaptability to tested environments. The genotype (BMZ 15-16-6) with more number of grains/spike (56.83), $b_{i}=1$ and nonsignificant for $S^{2}$ di value indicated that suitability in all types of environments similar report obtained by El-Morshidy (2001) and Abdel-Majeed (2005).

Whereas, the genotype UAS 304 recorded above average mean value for this character with $b_{i}>1$ indicating that these are sensitive to environmental changes but adapted to favourable environments (Table 2-5). 
Table.1 Analysis of variance for stability parameter of seed cotton yield and important yield components (Eberhert and Russell, 1966)

\begin{tabular}{|c|c|c|c|c|c|c|c|c|c|c|c|c|c|}
\hline Sources of variance & DF & $\begin{array}{l}\text { Days to } \\
\text { anthesis }\end{array}$ & $\begin{array}{l}\text { NDVI at } \\
\text { anthesis }\end{array}$ & $\begin{array}{c}\text { CT at } \\
\text { anthesis }\end{array}$ & $\begin{array}{l}\text { Chlorophyll } \\
\text { at anthesis }\end{array}$ & $\begin{array}{l}\text { days to } \\
\text { maturity }\end{array}$ & $\begin{array}{c}\text { spike } \\
\text { length } \\
\text { (cm) }\end{array}$ & $\begin{array}{c}\text { No. of } \\
\text { spiklets }\end{array}$ & tillers/m & $\begin{array}{c}\text { Grain yield } \\
(\mathrm{Kg} / \mathrm{ha})\end{array}$ & $\begin{array}{c}\text { Biomass } \\
(\mathrm{Kg} / \mathrm{ha})\end{array}$ & $\begin{array}{c}1000 \mathrm{GW} \\
\text { (g) }\end{array}$ & $\begin{array}{l}\text { Grain/ } \\
\text { Spike }\end{array}$ \\
\hline Varieties & 17 & $48.03 * * *$ & $0.003^{*}$ & $1.74 * *$ & $8.84 * *$ & $44.502 * *$ & $5.73 * * *$ & $9.68 * * *$ & $280.75^{* *}$ & $92362.18 * *$ & $548024.4 * * *$ & 5.567 & $69.84 * * *$ \\
\hline Env.+ (Var.* Env.) & 36 & 12.389 & $0.003^{* *}$ & $1.77 * *$ & $5.61 * *$ & $31.204^{* *}$ & 0.26 & 0.84 & 88.51 & $481782.8 * * *$ & $460191.8^{* * * *}$ & 16.535 & 16.181 \\
\hline Environments (Lin.) & 1 & $141.73 * * *$ & $0.094 * * *$ & $33.12 * * *$ & $43.29 * * *$ & $692.36 * * *$ & 0.41 & 0.47 & $456.23 *$ & $15751650.00^{* * * *}$ & $12820730^{* * *}$ & $167.00 * * *$ & $232.62 * * *$ \\
\hline Var.* Env.(Lin.) & 17 & 9.39 & 0.001 & $1.28 *$ & 6.42 & 13.949 & 0.13 & 0.46 & 77.70 & $68702.910 *$ & $150379.1^{*}$ & 13.74 & 6.00 \\
\hline Pooled Deviation & 18 & $8.03 * * *$ & $0.001^{* *}$ & $0.49 * *$ & $2.74 * * *$ & $10.77 * * *$ & $0.39 * * *$ & $1.21 * * *$ & $78.28 * * *$ & $23588.08 * *$ & $66096.16^{* *}$ & $10.81 * * *$ & $13.76 * * *$ \\
\hline Pooled Error & 51 & 1.063 & 0 & 0.20 & 0.65 & 1.195 & 0.07 & 0.32 & 5.45 & 8697.12 & 39592.13 & 0.62 & 3.06 \\
\hline
\end{tabular}

Table.2 Estimates of stability parameters of individual genotypes for days to anthesis, NDVI at anthesis and CT anthesis

\begin{tabular}{|c|c|c|c|c|c|c|c|c|c|c|}
\hline \multirow[t]{2}{*}{ S.N } & \multirow[t]{2}{*}{ Genotypes } & \multicolumn{3}{|c|}{ Days to anthesis } & \multicolumn{3}{|c|}{ NDVI at anthesis } & \multicolumn{3}{|c|}{ CT at anthesis } \\
\hline & & $\operatorname{Mean}(\overline{\mathrm{X}})$ & $\mathbf{b}_{\mathbf{i}}$ & $\mathbf{S}_{\mathrm{di}}^{2}$ & $\operatorname{Mean}(\bar{X})$ & $\mathbf{b}_{\mathbf{i}}$ & $\mathbf{S}_{\text {di }}^{2}$ & $\operatorname{Mean}(\overline{\mathrm{X}})$ & $\mathbf{b}_{\mathbf{i}}$ & $\mathbf{S}_{\mathrm{di}}^{2}$ \\
\hline 1 & UAS 347 & 62.50 & -1.134 & $4.325^{*}$ & 0.59 & 0.869 & 0.0004 & 25.46 & 1.993 & -0.064 \\
\hline 2 & NI 5439 & 69.16 & 1.255 & -0.776 & 0.56 & 1.343 & -0.0003 & 26.91 & 1.88 & $1.358 *$ \\
\hline 3 & GW 322 & 67.833 & 2.07 & 0.374 & 0.59 & 0.993 & 0.0001 & 26.60 & 1.217 & -0.026 \\
\hline 4 & UAS 304 & 71.16 & 1.837 & $7.055^{* *}$ & 0.59 & 1.508 & 0.0003 & 26.21 & $1.72 * *$ & -0.239 \\
\hline 5 & HI 944 & 59.16 & -0.835 & $20.624 * * *$ & 0.64 & 1.097 & $0.0019 *$ & 25.11 & 1.362 & 0.351 \\
\hline 6 & NIAW 34 & 59.33 & -1.219 & $19.911 * * *$ & 0.62 & 0.756 & 0.0004 & 24.98 & -0.34 & $1.274 *$ \\
\hline 7 & HD 3090 & 69.50 & 1.461 & 0.158 & 0.60 & 0.504 & $0.0029 * *$ & 25.81 & 1.984 & 0.044 \\
\hline 8 & UAS 415 & 69.50 & 0.243 & -1.013 & 0.62 & 0.429 & 0.0000 & 26.53 & 1.399 & 0.082 \\
\hline 9 & UAS 428 & 68.83 & 2.07 & 0.374 & 0.58 & 0.951 & $0.0021 * *$ & 25.9 & 1.147 & -0.127 \\
\hline 10 & DDK 1025 & 70.50 & 1.49 & $9.961 * *$ & 0.67 & 0.924 & 0.0007 & 25.21 & -0.712 & 0.18 \\
\hline 11 & DDK 1029 & 69.50 & 1.265 & -0.141 & 0.67 & 0.802 & 0.0003 & 24.58 & 0.392 & 0.599 \\
\hline 12 & HW 1098 & 66.66 & 0.898 & 1.265 & 0.64 & 1.026 & 0.0003 & 26.45 & 0.544 & -0.139 \\
\hline 13 & BMZ 15-16-10 & 68.50 & 1.621 & 2.763 & 0.62 & 1.049 & 0.0001 & 24.86 & -0.238 & -0.212 \\
\hline 14 & BMZ 15-16-5 & 61.33 & 0.354 & $19.134 * * *$ & 0.62 & 0.958 & 0.0003 & 24.65 & 0.466 & 0.206 \\
\hline 15 & BMZ 15-16-9 & 69.83 & 1.435 & $28.901 * * *$ & 0.62 & 1.132 & -0.0003 & 25.16 & 1.339 & -0.177 \\
\hline 16 & BMZ 15-16-2 & 68.16 & 1.077 & -0.006 & 0.60 & $1.446 * *$ & -0.0003 & 25.16 & 1.628 & 0.615 \\
\hline 17 & BMZ 15-16-7 & 69.66 & 1.92 & -0.897 & 0.63 & 0.591 & -0.0001 & 26.81 & 0.593 & $1.066^{*}$ \\
\hline 18 & BMZ 15-16-6 & 72.16 & 2.193 & $13.748 * * *$ & 0.60 & 1.621 & 0.0010 & 25.78 & 1.626 & -0.233 \\
\hline \multicolumn{2}{|c|}{ Population mean } & 67.40 & & & 0.61 & & & 25.68 & & \\
\hline
\end{tabular}


Table.3 Estimates of stability parameters of individual genotypes for chlorophyll at anthesis, days to maturity and spike length (cm)

\begin{tabular}{|c|c|c|c|c|c|c|c|c|c|c|}
\hline \multirow[t]{2}{*}{ S.N } & \multirow[t]{2}{*}{ Genotypes } & \multicolumn{3}{|c|}{ Chlorophyll at anthesis } & \multicolumn{3}{|c|}{ Days to maturity } & \multicolumn{3}{|c|}{ Spike length (cm) } \\
\hline & & $\operatorname{Mean}(\overline{\mathrm{X}})$ & $\mathbf{b}_{\mathbf{i}}$ & $\mathbf{S}_{\mathrm{di}}^{2}$ & $\operatorname{Mean}(\overline{\mathrm{X}})$ & $\mathbf{b}_{\mathbf{i}}$ & $\mathbf{S}_{\mathrm{di}}^{2}$ & $\operatorname{Mean}(\overline{\mathrm{X}})$ & $\mathbf{b}_{\mathbf{i}}$ & $\mathbf{S}_{\mathrm{di}}^{2}$ \\
\hline 1 & UAS 347 & 49.75 & 0.091 & 1.028 & 89.33 & 0.262 & $12.322 * *$ & 8.15 & 1.917 & -0.042 \\
\hline 2 & NI 5439 & 51.45 & -0.335 & -0.603 & 96.00 & 0.814 & $9.79 * *$ & 7.26 & 2.765 & $0.69 * *$ \\
\hline 3 & GW 322 & 48.56 & 3.818 & -0.503 & 91.33 & 1.181 & $9.833 * *$ & 8.26 & 0.472 & -0.023 \\
\hline 4 & UAS 304 & 48.6 & 2.621 & $2.688 *$ & 98.33 & 1.89 & $7.52 * *$ & 9.12 & -0.168 & -0.063 \\
\hline 5 & HI 944 & 48.36 & -0.9 & $4.585^{* *}$ & 86.00 & -0.018 & -0.721 & 7.26 & -0.809 & 0.008 \\
\hline 6 & NIAW 34 & 50.10 & -1.111 & 0.629 & 86.00 & $0.107 *$ & -1.145 & 7.23 & 0.267 & 0.021 \\
\hline 7 & HD 3090 & 49.73 & 1.539 & 1.269 & 95.66 & 0.926 & $15.963 * * *$ & 8.20 & -0.468 & 0.071 \\
\hline 8 & UAS 415 & 53.08 & -2.543 & -0.061 & 94.83 & 0.419 & 2.21 & 6.31 & 8.182 & $0.799 * * *$ \\
\hline 9 & UAS 428 & 50.12 & 1.289 & -0.428 & 94.33 & 0.58 & -0.961 & 5.10 & -1.812 & $0.402 * *$ \\
\hline 10 & DDK 1025 & 51.15 & 1.059 & $1.952 *$ & 97.16 & 1.818 & $49.821 * * *$ & 7.90 & 2.478 & $2.817 * * *$ \\
\hline 11 & DDK 1029 & 52.73 & $3.711 *$ & -0.613 & 96.50 & 1.865 & $39.504 * * *$ & 8.55 & 2.385 & $0.203 *$ \\
\hline 12 & HW 1098 & 51.30 & 2.572 & $20.488 * * *$ & 91.50 & 1.063 & 0.796 & 8.43 & -0.275 & $0.661 * *$ \\
\hline 13 & BMZ 15-16-10 & 54.30 & 1.876 & 0.452 & 94.50 & 0.993 & -1.135 & 10.10 & 0.604 & -0.012 \\
\hline 14 & BMZ 15-16-5 & 48.98 & 1.149 & 0.76 & 88.16 & 0.84 & -1.151 & 8.15 & 2.86 & -0.063 \\
\hline 15 & BMZ 15-16-9 & 52.97 & 0.855 & -0.024 & 97.33 & 1.715 & 2.306 & 10.43 & -0.941 & -0.037 \\
\hline 16 & BMZ 15-16-2 & 49.65 & 0.73 & $4.102 * *$ & 91.66 & 0.962 & $6.34 *$ & 10.55 & -2.114 & $0.57 * *$ \\
\hline 17 & BMZ 15-16-7 & 50.88 & 1.275 & 0.321 & 93.50 & 0.921 & $8.181 * *$ & 9.25 & 0.37 & -0.062 \\
\hline 18 & BMZ 15-16-6 & 50.98 & 0.306 & $1.943^{*}$ & 96.33 & 1.663 & $12.609 * *$ & 8.83 & 2.289 & -0.056 \\
\hline \multicolumn{2}{|c|}{ Population mean } & 50.70 & & & 93.25 & & & 8.28 & & \\
\hline
\end{tabular}


Table.4 Estimates of stability parameters of individual genotypes for Number of spikelets, no. tiller/m and grain yield (Kg/ha)

\begin{tabular}{|c|c|c|c|c|c|c|c|c|c|c|}
\hline \multirow[t]{2}{*}{ S.N } & \multirow[t]{2}{*}{ Genotypes } & \multicolumn{3}{|c|}{ Number of spikelets } & \multicolumn{3}{|c|}{ No. Tillers/m² } & \multicolumn{3}{|c|}{ Grain yield (Kg/ha) } \\
\hline & & $\operatorname{Mean}(\bar{X})$ & $\mathbf{b}_{\mathbf{i}}$ & $\mathbf{S}_{\text {di }}^{2}$ & $\operatorname{Mean}(\overline{\mathrm{X}})$ & $\mathbf{b}_{\mathbf{i}}$ & $\mathbf{S}_{\text {di }}^{2}$ & $\operatorname{Mean}(\overline{\mathrm{X}})$ & $\mathbf{b}_{\mathbf{i}}$ & $\mathbf{S}_{\text {di }}^{2}$ \\
\hline 1 & UAS 347 & 17.56 & 3.77 & -0.163 & 68.66 & 1.547 & $45.588^{* *}$ & 2618.33 & 0.938 & $82760.102 * *$ \\
\hline 2 & NI 5439 & 18.93 & 4.169 & -0.286 & 76.83 & -1.555 & $33.985^{*}$ & 3070.00 & $1.281^{*}$ & -10101.34 \\
\hline 3 & GW 322 & 22.06 & -4.768 & -0.185 & 78.66 & 3.099 & $211.916 * * *$ & 3162.00 & 1.231 & 14466.395 \\
\hline 4 & UAS 304 & 20.83 & -4.893 & 0.204 & 94.00 & 0.081 & $228.437 * * *$ & 3129.00 & $1.293 * *$ & -10149.625 \\
\hline 5 & HI 944 & 17.60 & 6.816 & $1.413^{*}$ & 69.33 & 0.673 & $97.786^{* * *}$ & 2747.83 & $0.69 *$ & -10050.722 \\
\hline 6 & NIAW 34 & 16.96 & -0.125 & 0.845 & 65.00 & -2.161 & $146.277 * * *$ & 2965.67 & 0.99 & -10060.895 \\
\hline 7 & HD 3090 & 20.40 & 2.846 & -0.271 & 72.33 & 1.611 & -5.53 & 2804.17 & 0.97 & 4855.796 \\
\hline 8 & UAS 415 & 18.83 & 10.311 & $1.165^{*}$ & 78.66 & 0.866 & $18.277^{*}$ & 3038.33 & 1.28 & 6874.943 \\
\hline 9 & UAS 428 & 17.80 & 3.745 & 0.055 & 83.33 & 1.539 & $176.702 * * *$ & 2662.17 & 0.711 & $32333.943 *$ \\
\hline 10 & DDK 1025 & 21.61 & -3.383 & $2.213 * *$ & 74.33 & 3.419 & 13.991 & 2892.83 & 1.637 & 26902.873 \\
\hline 11 & DDK 1029 & 22.43 & -3.545 & $5.159 * * *$ & 82.66 & 3.912 & 0.925 & 3090.17 & 1.173 & -6040.389 \\
\hline 12 & HW 1098 & 19.38 & 3.308 & $1.996 * *$ & 82.33 & 1.242 & -0.356 & 3131.50 & 1.016 & 5131.91 \\
\hline 13 & BMZ 15-16-10 & 21.06 & -1.398 & 0.055 & 62.50 & -1.732 & 14.105 & 2901.67 & 0.802 & 16966.899 \\
\hline 14 & BMZ 15-16-5 & 18.76 & 3.245 & 0.072 & 72.50 & 1.343 & $35.919 *$ & 3014.17 & 0.734 & 5538.952 \\
\hline 15 & BMZ 15-16-9 & 21.63 & -1.448 & $3.011 * *$ & 94.16 & 0.164 & 16.585 & 3255.33 & 1.043 & $121174.382 * * *$ \\
\hline 16 & BMZ 15-16-2 & 22.30 & -3.52 & 0.937 & 91.83 & -0.301 & -2.023 & 3037.67 & 0.991 & -9253.291 \\
\hline 17 & BMZ 15-16-7 & 21.13 & 1.248 & 0.145 & 71.33 & 3.215 & $20.356^{*}$ & 2990.17 & $0.59 *$ & -9943.305 \\
\hline 18 & BMZ 15-16-6 & 20.83 & 1.623 & -0.223 & 66.16 & 1.038 & $249.977 * * *$ & 2859.50 & $0.63 *$ & -9603.431 \\
\hline \multicolumn{2}{|c|}{ Population mean } & 20.00 & & & 76.92 & & & 2965.02 & & \\
\hline
\end{tabular}


Table.5 Estimates of stability parameters of individual genotypes for Biomass (Kg/ha), 1000 Grain Weight (g) and Grain/ spike

\begin{tabular}{|c|c|c|c|c|c|c|c|c|c|c|}
\hline \multirow[t]{2}{*}{ S.N } & \multirow[t]{2}{*}{ Genotypes } & \multicolumn{3}{|c|}{ Biomass (Kg/ha) } & \multicolumn{3}{|c|}{1000 Grain Weight (g) } & \multicolumn{3}{|c|}{ Grain/ spike } \\
\hline & & Mean $(\bar{X})$ & $\mathbf{b}_{\mathbf{i}}$ & $\mathbf{S}_{\mathrm{di}}^{2}$ & Mean $(\overline{\mathrm{X}})$ & $\mathbf{b}_{\mathbf{i}}$ & $\mathbf{S}_{\mathrm{di}}^{2}$ & $\operatorname{Mean}(\overline{\mathrm{X}})$ & $\mathbf{b}_{\mathbf{i}}$ & $\mathbf{S}_{\text {di }}^{2}$ \\
\hline 1 & UAS 347 & 4224.83 & 1.574 & $176654.997 *$ & 40.00 & 1.506 & $33.797 * * *$ & 54.16 & 0.793 & -0.877 \\
\hline 2 & NI 5439 & 5660.67 & 1.237 & -27932.936 & 40.43 & 2.142 & $6.127 * *$ & 52.33 & 0.402 & 7.154 \\
\hline 3 & GW 322 & 5611.83 & 1.129 & -20301.284 & 41.16 & 3.254 & $3.481 *$ & 54.33 & 0.403 & $12.143 *$ \\
\hline 4 & UAS 304 & 5519.00 & 1.398 & 24950.999 & 41.13 & 2.14 & -0.144 & 51.83 & 1.587 & 1.712 \\
\hline 5 & HI 944 & 4792.83 & 0.886 & -20091.641 & 40.86 & 0.459 & $32.267 * * *$ & 49.00 & 1.189 & 3.32 \\
\hline 6 & NIAW 34 & 5192.00 & 0.884 & -19889.289 & 39.83 & 0.906 & -0.135 & 48.33 & 0.393 & 7.253 \\
\hline 7 & HD 3090 & 4823.67 & 1.064 & 46344.93 & 41.68 & 1.054 & -0.524 & 53.00 & 2.377 & $22.044 * *$ \\
\hline 8 & UAS 415 & 5528.83 & 1.451 & -25125.173 & 42.25 & -0.001 & -0.197 & 50.66 & 2.826 & $70.026 * * *$ \\
\hline 9 & UAS 428 & 5082.17 & 0.912 & -31463.045 & 39.15 & 0.208 & $5.627 * *$ & 44.33 & 0.734 & 3.275 \\
\hline 10 & DDK 1025 & 4792.83 & 1.87 & $154466.576^{*}$ & 40.15 & $-0.877^{*}$ & -0.515 & 43.16 & 0.964 & -1.756 \\
\hline 11 & DDK 1029 & 5591.33 & 1.1 & -38048.299 & 40.35 & -1.27 & $8.54 * * *$ & 49.33 & 1.425 & $11.987 *$ \\
\hline 12 & HW 1098 & 5637.83 & 1.159 & -11342.185 & 42.68 & 1.174 & -0.104 & 47.33 & 1.078 & -2.781 \\
\hline 13 & BMZ 15-16-10 & 5388.17 & 1.039 & -24616.196 & 43.06 & 1.192 & 1.432 & 59.83 & 0.574 & $14.984^{*}$ \\
\hline 14 & BMZ 15-16-5 & 5888.17 & 0.846 & -34855.891 & 41.16 & -0.6 & $5.674 * *$ & 52.66 & 0.626 & -1.822 \\
\hline 15 & BMZ 15-16-9 & 5783.00 & 0.67 & $348853.301 * *$ & 41.71 & 1.092 & -0.591 & 62.16 & 0.465 & $46.951 * * *$ \\
\hline 16 & BMZ 15-16-2 & 5269.50 & 0.492 & -37160.584 & 37.75 & 1.834 & $5.397 * *$ & 52.50 & 0.684 & -0.959 \\
\hline 17 & BMZ 15-16-7 & 5490.50 & 0.412 & -39967.628 & 42.53 & 2.817 & $20.945^{* * *}$ & 49.33 & 0.567 & -2.405 \\
\hline 18 & BMZ 15-16-6 & 5306.50 & -0.122 & 8946.96 & 42.28 & 0.971 & $62.868 * * *$ & 56.83 & 0.913 & 4.978 \\
\hline \multicolumn{2}{|c|}{ Population mean } & 5310.20 & & & 41.07 & & & 51.73 & & \\
\hline
\end{tabular}


The present study suggest that October 25 is the most optimum time of planting of wheat crop, because the crop sown on October 25 produced the maximum grain yield, number of tillers per meter row and grains per spike. The rate of reduction after October 25 planting for grain yield, number of grain per spike, 1000 grain weight and number of tiller per meter row. Similar findings were reported by earlier research workers Chaudhry et al., (1995), Iqbal et al., (2001) and Ahmad et al., (1996). The wheat variety BMZ $15-16-2$ is most stable for grain yield, tillers $/ \mathrm{m}^{2}$, number of spikelets, grains per spike, early maturity and spike length and HW 1098 most stable for tillers $/ \mathrm{m}^{2}, 1000$ grain weight, high biomass and days to maturity.

\section{References}

Abdel-Majeed SA, Mousa AM, Abd ElKareem AA (2005). Effect of heat stress on some agronomic of bread wheat [Triticum aestivum] genotypes under Upper Egypt conditions. Fayoum. $J$. Agric. Res. Dev. 19(1):4-16.

Ahmad, J., Chaudhry, M.H., Din, S. and Ali, M.A. (1996). Stability for grain yield in wheat. Pakistan J. Bot., 28: 61-5

Al-Otayk, M. S. (2010). Performance of Yield and Stability of Wheat Genotypes under High Stress Environments of the Central Region of Saudi Arabia. Met., Environment \& Arid Land Agriculture Science, 21(1): 81-92.

Arain, A. M., Sial, A. M., Rajput, A. M. and Mirbahar, A. A. (2011). Yield stability in bread wheat genotypes. Pakistan J. Bot, 43(4): 2071-2074.

Aydin, N., Bayramoglu, H. O. and Ozcan, F.L. (2010). Stability of some quality traits in bread wheat (Triticum aestivum) genotypes. Journal of Environment Biology, 31: 489-95.

Chaudhry, M.H., Anwar, J., Hussain, F. and Khan, F.A. (1995). Effect of planting time on grain yield in wheat varieties. $J$. Agric. Res., 33: 103-8.

Eberhart, S. A. and Russel, R. A. (1966). Stability parameters for comparing varieties. Crop Sci., 6: 36-40.

El-Morshidy MA, Kherialla KA, Abdel-Ghan AM, Abdel -Kareem (2001). Stability analysis for earliness and grain yield in bread wheat the 2 plant Breed comp. October 2, Assiut, University, pp. 199 -217.

Gowda, D., Singh, S.S., Singh, G.P., Deveshwar, A.M., and Ahlawat, A. (2010). Stability analysis for physiological and quality parameters in wheat (Triticum aestivum). Indian J. of Agri. Sci, 80(12):102832.

Gulzar, S., Kamaluddin, Bhatt, M. A., Yusuf, N. and Khan, M. A. (2015). Stability analysis for yield, yield component and quality traits in wheat (Triticum aestivum L.) under temperate conditions in kashmir valley. Plant Archives., 15: 433-440.

IIWBR, (2016). Annual Report of Indian Institute of wheat and barley Research.

Iqbal, M.S., Yar,A., Ali, A., Anser, M.R., Iqbal, J. and Akram, M.H. (2001). Effect of sowing dates and seed rate on grain yield of wheat (CV.93-BT-022). J. Agric. Res., 39: 217-21.

Kant, S., Lamba, R. A. S, Arya, R.K. and Panwar, I.S. (2014). Effect of terminal heat stress on stability of yield and quality parameters in bread wheat in south-west Haryana. J. of Wheat Res., 6(1):64-73.

Kashte S. (2013) "Stability analysis for yield and quality traits in wheat" (Triticum aestivum L.) Thesis submitted to V.N.M.K.V. Parbhani.

Kaya N, Paita C, Taner S (2002). Additive main effects and multiplicative interaction analysis of yield performance in bread wheat genotypes across environment. Turk. J. Agric. 26:275-279. 
Kirgiwi, F.M., Ginkel, M. V., Trethowan, R., Sears, R. G., Rajaram, S. and Paulsen, G. M. (2004) Evaluation of selection strategies for wheat adaptation across water regimes. Euphytica, 135: 361-71.

Kumar, V., Tyagi, B.S., Verma, A. and Sharma, I. (2014). Stability analysis for grain yield and its components under different moisture regimes in bread wheat (Triticum aestivum). Indian J. of AgriSci, 84(8): 931-6.

Lodhi, R. D., Prasad, L. C., Bornare, S. S., Madakemohekar, A. H., And Prasad, R.(2015). Stability analysis of yield and its component traits of barley (Hordeum vulgare 1.) genotypes in multienvironment trials in the north eastern plains of india. $J$. of Breeding and Genetics., 47(2): 143-159.

Meena, H.S., Kumar. D,, Srivastava, T.K. and Rajendra, P.S. (2014). Stability for grain yield and its contributing traits in bread wheat (Triticum aestivum). Indian J. of AgriSci, 84(12): 1486-95.

Menshawy, A.M.M. (2007) Evaluation of some early bread wheat genotypes under different sowing dates: 1 Earliness characters. Fifth plant breeding conference (May), Egypt J. Plant Breed, 11(1): 25-40.

Motamedi, M., Naserierad, H., Naseri, R. and Soleymanifard, A. (2012). Assessment of genotype $\times$ environment interaction for grain yield in bread wheat genotypes. Intl J. of Agri Crop Sci., 4(18): 1366-1370.

Sharma, C. R., Morgounov, I. A., Braun, J. H., Akin, B., Keser, M., kaya, Y.,
Khalikulov, Z., Ginkel, M., Yahyaoui, A. and Rajaram, S. (2012). Yield stability analysis of winter wheat genotypes targeted to semi-arid environments in the international winter wheat improvement program. International $J$. of Plant Breeding, 6(1):7-13.

Sial, A. M, Arain, A. M. and Ahmad, M. (2000). Genotype x environment interaction on bread wheat grown over multiple sites and years in Pakistan. Pakistan J. Bot, 32(1) 85-91.

Singh, T. and Tyagi, J.P. (2014). Stability for Yield and its Component Traits in Durum Wheat (Triticum durum Desf.). Indian J. of Plant Genetic Res, 27 (3): 287-94.

Swami, A. 2012. Stability analysis for different quantitative and physiological traits in bread wheat. M.Sc. thesis. Sam Higginbottom University of Agriculture, Technology and Sciences, Allahabad, India.

Thakare, S. D., Potdukhe, N. R. and Bharad, S. G (2014). Yield Stability over Sowing Windows in Wheat. $J$. of pkvresearch., 38(2): 30-36.

Yadav, R. and Singh, T. B. (2003). Stability analysis in wheat for grain protein. Indian J. of Genetics., 63(4): 337-338.

Yan, W., Fregeau-Reid, J., Pageau, D., Martin, R., MitchellFetch, J., Etienne, M., Rowsell, J., Scoot, P., Price, M., Haan, B., Cummiskey -Alajeunesse, J., Durand, J., Sparry, E. (2010). Identifying essential test locations for oat breeding in eastern Canada. Crop Sci, 60: 504-515.

\section{How to cite this article:}

Santoshkumar Pujer, V. Rudra Naik, Suma S. Biradar and Uday, G. 2020. Stability Analysis to Study the Effects of Different Date of Sowing on Grain Yield Performance in Wheat (Triticum sp.). Int.J.Curr.Microbiol.App.Sci. 9(05): 2343-2353.

doi: https://doi.org/10.20546/ijcmas.2020.905.267 